\title{
Cost-effectiveness analysis of orbital atherectomy plus balloon angioplasty vs balloon angioplasty alone in subjects with calcified femoropopliteal lesions
}

\author{
This article was published in the following Dove Press journal: \\ ClinicoEconomics and Outcomes Research \\ 19 March 2014 \\ Number of times this article has been viewed
}

\section{Barry Weinstock' \\ Raymond Dattilo² \\ Tiffini Diage ${ }^{3}$}

'Orlando Health Heart Institute, Mid-Florida Cardiology Specialists, Orlando, FL, USA; ${ }^{2}$ Department of Cardiology, St Francis Health Center, Topeka, KS, USA; ${ }^{3}$ North American Science Association (NAMSA),

Sunnyvale, CA, USA

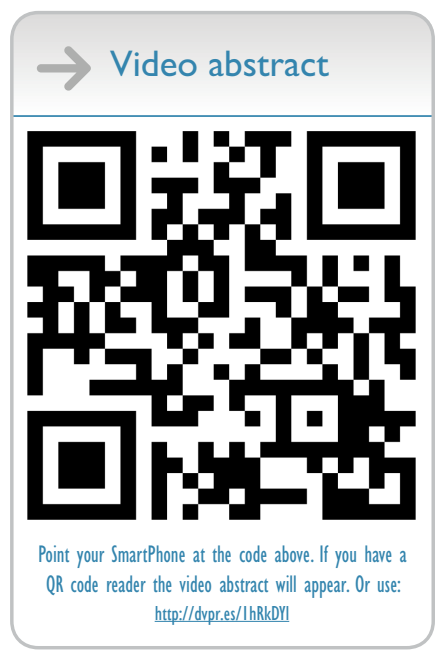

Correspondence: Tiffini Diage Medical Research Manager, North American Science Association (NAMSA), I 307 S Mary Avenue, Sunnyvale, CA 94087, USA

$\mathrm{Tel}+\mathrm{I} 7077996732$

$\mathrm{Fax}+16509671903$

Email tdiage@namsa.com
Introduction: As cost considerations become increasingly critical when selecting optimal endovascular treatment strategies, a cost-benefit analysis was conducted comparing the Diamondback $360^{\circ \otimes}$ Orbital Atherectomy System (OAS) (Cardiovascular Systems, Inc., St Paul, MN, USA) and balloon angioplasty (BA) vs BA alone for treatment of calcified femoropopliteal lesions.

Patients and methods: The clinical outcomes from COMPLIANCE $360^{\circ}$, a prospective, multicenter, randomized study comparing OAS+BA vs BA alone for treatment of calcified femoropopliteal lesions, were correlated with cost data and previously published quality of life data. Site of service, hospital charges, and associated medical resource utilization were obtained from Uniform Billing statements for index treatments and associated revascularizations out to 1 year. Hospital costs were estimated using hospital-specific, procedure-specific cost-to-charge ratios. Length of stay and procedural data were collected from participating study sites.

Results: Twenty-five subjects with 38 lesions and 25 subjects with 27 lesions were randomized to the OAS+BA and BA-alone groups, respectively. Mean hospital charges (US\$51,755 vs US $\$ 39,922$ ) and estimated hospital costs (US\$15,100 vs US\$11,016) were higher for OAS+BA compared with BA alone (not statistically significant). Stent utilization was statistically significantly higher with BA-alone treatment for all subjects $(1.1$ vs $0.1, P=0.001)$ and in the subset of subjects with one lesion $(1.0 \mathrm{vs} 0.1, P<0.00001)$. There was a significant difference in cost for single-lesion versus multiple-lesion treatment. Using costs and quality-adjusted life years (QALYs) for the single-lesion cohort, the 1-year incremental cost of OAS+BA vs BA alone was US\$549, and incremental QALY was 0.16 . This results in an incremental cost-effectiveness ratio of US $\$ 3,441$, well below the US\$50,000 threshold.

Conclusion: One-year index procedure cost and cost-effectiveness were comparable for OAS+BA vs BA alone. This study provides compelling cost-effectiveness data for using atherectomy for treatment of calcified femoropopliteal lesions, a longstanding challenge for peripheral artery disease interventionalists.

Keywords: peripheral vascular disease, orbital atherectomy, cost analysis

\section{Introduction}

Peripheral artery disease (PAD) affects 12\%-20\% of Americans 60 years of age and older. ${ }^{1,2}$ Although balloon angioplasty (BA) is typically the first-line revascularization strategy, intermediate and long-term outcomes are uniformly poor for all but the simplest TransAtlantic Inter-Society Consensus type A lesions, with rates of restenosis ranging from $40 \%$ to $60 \%$ at 1 year. ${ }^{3,4}$ American College of Cardiology/American Heart Association guidelines currently recommend against primary stenting of 
femoropopliteal lesions (class III indication). ${ }^{3}$ Remarkably, atherectomy devices as well as stenting are indicated (class IIb) as "salvage" devices to be used in the event of suboptimal BA results. ${ }^{3}$ The presence of calcified plaque in femoropopliteal lesions is common, and arterial wall calcium is associated with higher rates of procedural complications, including flow-limiting dissections which frequently require stent deployment to maintain vessel patency. ${ }^{5,6}$ Further, stenting as salvage therapy in calcified segments after failed BA often results in stent underexpansion and malapposition, which increase the probability of subsequent stent fracture and/or restenosis. ${ }^{7,8}$ Lastly, diffuse pattern in-stent restenosis (ISR) has no approved treatment modality and is often a harbinger for multiple reinterventions or definitive bypass surgery. Biomechanical stresses across the dynamic femoropopliteal segment further add to the complexity of realizing successful long-term clinical outcomes.

Lesion modification utilizing atherectomy prior to percutaneous transluminal angioplasty (PTA) or stent implantation may be recommended as an alternative treatment option in patients with severely calcified lesions. The Orbital Atherectomy System (OAS) (Cardiovascular Systems, Inc., St Paul, MN, USA) is a minimally invasive catheter-based device that may be safely utilized for revascularization of occluded peripheral vessels. ${ }^{9-11}$ For example, the OAS device has been used to effectively modify severely calcified infrapopliteal lesions prior to BA by changing vessel compliance, resulting in fewer dissections, less bailout stenting, and lower adjunctive balloon pressure to achieve the desired angioplasty result. ${ }^{12}$ A recent study evaluating modification of calcified lesions in above-the-knee, below-the-knee, and popliteal arteries utilizing the OAS device found an elimination of bailout stenting, improved patency, and low reintervention rates. ${ }^{11}$

Despite the recent advances in endovascular therapy, costeffective methods of care have not been well defined. The direct and indirect costs of cardiovascular and stroke-related care in the US in 2008 were estimated to be US $\$ 448.5$ billion and represented the largest disease costs to Medicare. ${ }^{13}$ Mean Medicare expenditures in 2001 for PAD were US\$13,901 or more than double the average expenditure per enrollee (US\$5,833). ${ }^{14}$ However, little is known about the actual costs associated with different PAD interventions among patients with calcified lesions. Understanding the economic impact of care for this patient population is vital. Using data from the COMPLIANCE $360^{\circ}$ randomized pilot study, we conducted a cost-effectiveness analysis comparing OAS with adjunctive BA vs BA alone.
Utilizing a threshold of US $\$ 50,000$ per quality-adjusted life year (QALY), the objective of the study is to determine whether orbital atherectomy is a cost-effective alternative for treatment of PAD. The study was performed from the third-party payer perspective and is intended for policymakers weighing decisions regarding technology adoption.

\section{Methods \\ Study design}

COMPLIANCE $360^{\circ}$, a prospective study, was conducted to compare the performance of the OAS followed by BA (OAS+BA) vs BA alone at nine US centers. Subjects with Rutherford class 2-4 classification had lesions of $\geq 70 \%$ stenosis and fluoroscopically visible calcium and were equally randomized to each arm of the study after meeting all inclusion criteria and no exclusion criteria. The COMPLIANCE $360^{\circ}$ study design has been previously described. ${ }^{15}$

\section{Economic endpoints}

$\mathrm{OAS}+\mathrm{BA}$ and BA procedure charges, procedure time, length of stay (LOS), and stent and balloon utilization were collected for enrolled subjects. The following data were collected for the index procedures and associated hospitalizations from the subjects' Uniform Billing statements (UB-04s): hospital charges, site of service (inpatient or outpatient setting), and associated medical resource utilization (MRU). LOS and procedural data (procedure time, number of lesions treated, stents utilized, and balloons utilized) were collected from the participating COMPLIANCE $360^{\circ}$ study sites.

Hospital costs were estimated from hospital charges using hospital-specific, procedure-specific cost-to-charge ratios (CCRs) obtained through the American Hospital Directory (http://www.ahd.com) and based on the hospital's most recent Medicare cost report. All charges and costs are reported in 2010 US dollars. Each patient's UB-04 charges for the entire procedure report were used to calculate the estimated costs based on reported inpatient Diagnosis-Related Group (DRG) billing code or hospital outpatient International Classification of Diseases, Ninth Revision (ICD-9) diagnostic code. For inpatient procedures, the average among the three hospitals that specifically reported DRGs 252, 253, and 254 was used to calculate the average CCR. For hospital outpatient procedures, the hospital-specific CCR was calculated from the reported average cost and charge listed for the ICD-9 diagnostic codes from 440.2x; Ambulatory Payment Classification codes were not reported. 


\section{Data analysis}

A decision tree was constructed based on procedural success and outcomes through 1 year (Figure 1). These endpoints are defined as patency, restenosis of the treated vessel (defined as reported target lesion revascularization [TLR] or target vessel revascularization [TVR]), and claudication with no subsequent treatment. Since more subjects in the OAS arm had multiple lesions requiring treatment, the decision tree and cost-effectiveness analysis compared the subset of subjects with only one lesion treated. Primary and secondary clinical study results are presented alongside cost and QALY data to compare the index procedure costs and longer-term (1-year) comparative effectiveness of OAS+BA vs BA alone.

For this analysis, we defined procedural success as freedom from adjunctive stenting. Procedural success and failure were considered separately for each arm. The average cost of reintervention for each arm was estimated based on the types of interventions performed (surgical bypass, stenting, or BA) and the hospital-specific CCRs for those interventions. Restenosis requiring intervention evaluated by duplex ultrasound and claudication without intervention were both considered separately for each procedure arm. Periprocedural and acute adverse events (pseudoaneurysm and embolic event) were included in procedure costs.

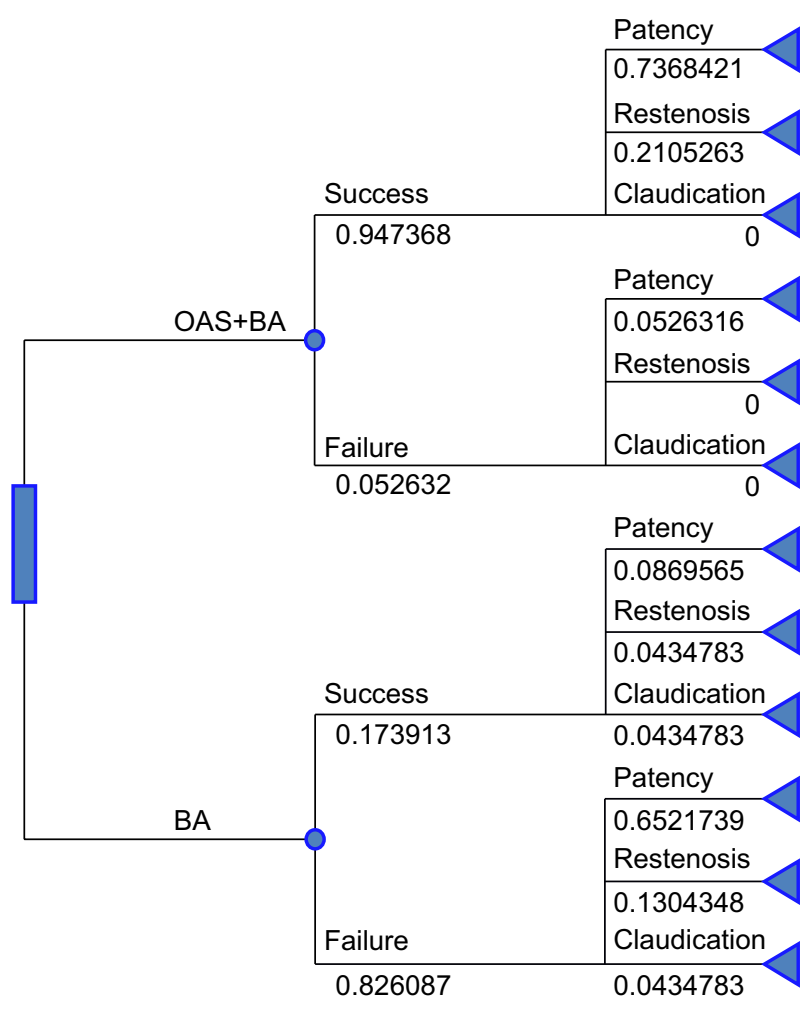

Figure I Decision tree and proportional outcomes (single-lesion population). Abbreviations: BA, balloon angioplasty; OAS, orbital atherectomy system.
QALYs were used from previously published literature. An incremental cost-effectiveness ratio was calculated based on proportional cost and QALY for each outcome in OAS+BA compared with BA alone.

Standard summary statistics were calculated for study variables of interest. For continuous variables, statistics included the number of observations $(\mathrm{N})$, mean, standard deviation, and $95 \%$ confidence intervals. A linear regression was conducted to determine associations between cost and other variables. Statistical analyses were conducted in SAS version 9.3 (SAS Institute, Cary, NC, USA).

\section{Results}

Twenty-five subjects with 38 lesions and 25 subjects with 27 lesions were randomized to receive OAS+BA and BA alone, respectively. Prevalence of diabetes was significantly higher $(P<0.05)$ in the OAS+BA arm $(72 \%)$ than in the BA-alone arm (40\%). The number of lesions per patient (1.5 vs $1.1, P<0.05)$ and number of lesions with single-vessel runoff ( 18 vs $3, P<0.05$ ) were also significantly higher in the OAS+BA arm, while mean percent stenosis was significantly lower $(83.9 \%$ vs $92.8 \%, P=0.003)$. There were no other statistically significant differences in patient demographics, comorbidities, medical history, lesion characteristics, plaque morphology, or calcium scores.

In the OAS+BA study arm, 17 outpatient and eight inpatient procedures were performed, with two of the inpatient procedures performed on subjects with complications and/or comorbidities (DRG code 253) and an additional two on subjects with major complications and/or comorbidities (DRG code 254). In the BA arm, 19 procedures were performed on an outpatient basis, with the six remaining procedures resulting in an inpatient hospital stay. Of the six inpatient procedures, two were for subjects with diagnostic codes indicating procedural complications and/or patient comorbidities. Table 1 provides summary statistics regarding number of lesions treated as well as intra- and post-procedure MRU as specified above.

Despite the statistically higher lesion counts, significantly fewer stents ( 0.1 vs 1.1 per person) were utilized during the OAS+BA procedures, while mean balloon usage was comparable between treatment groups. Mean hospital charges (US\$51,755 vs US\$39,922) and estimated hospital costs (US $\$ 15,100$ vs US $\$ 11,016$ ) were higher for OAS+BA compared with BA alone, but the difference was not statistically significant. Mean procedure time was significantly longer (96 vs 69 minutes, $P<0.005$ ) in the OAS+BA treatment group, while the mean patient LOS was 
Table I Comparison of medical resource utilization for index procedure (all subjects)

\begin{tabular}{|c|c|c|c|c|c|}
\hline & \multicolumn{2}{|l|}{ OAS+BA $(n=25)$} & \multicolumn{2}{|l|}{ BA $(n=25)$} & \multirow[t]{2}{*}{$P$-value } \\
\hline & Mean \pm SD & Range & Mean \pm SD & Range & \\
\hline Number of lesions (per patient) & $1.5 \pm 1.2$ & $1-6$ & $1.1 \pm 0.3$ & $1-2$ & 0.05 \\
\hline Procedure time $(\mathrm{min})$ & $96 \pm 41$ & $34-179$ & $69 \pm 17$ & $37-106$ & 0.005 \\
\hline Stents utilized (per patient) & $0.1 \pm 0.3$ & $0-1$ & $1.1 \pm 0.8$ & $0-3$ & 0.001 \\
\hline Balloons utilized (per patient) & $1.7 \pm 1.2$ & $0-6$ & $1.6 \pm 0.9$ & $1-4$ & NS \\
\hline Hospital charge $^{a}$ & $\$ 51,755 \pm \$ 23,852$ & $\$ 12,676-\$ 124,161$ & $\$ 39,922 \pm 15,187$ & $\$ 12,36 \mid-\$ 67,226$ & NS \\
\hline Estimated hospital cost $\mathrm{t}^{\mathrm{a}, \mathrm{b}}$ & $\$ 15,100 \pm \$ 8,501$ & $\$ 5,390-\$ 40,245$ & $\$ 11,016 \pm \$ 5,675$ & $\$ 5,257-\$ 31,077$ & NS \\
\hline LOS (hours) ${ }^{c}$ & $22.4 \pm 22.1$ & $2.1-97.3$ & $23.7 \pm 38.3$ & $4.4-180.8$ & NS \\
\hline
\end{tabular}

Notes: Dollar values are in USD. aPhysician charges are not included; bhospital costs were estimated from hospital charges by using hospital-specific and procedure-specific cost-to-charge ratios obtained from http:llwww.ahd.com; 'LOS data were available for 42 subjects (22 receiving OAS+BA and 20 receiving BA alone).

Abbreviations: BA, balloon angioplasty; LOS, length of stay; NS, not significant; OAS, orbital atherectomy system; SD, standard deviation.

not significantly different for OAS+BA (22.4 hours) vs BA alone (23.7 hours).

A linear regression analysis was fit to determine whether there was an association of cost and number of lesions treated. Because of the limited number of subjects with more than one lesion treated, two lesion cohorts were created: 1) one lesion treated and 2) more than one lesion treated. There was a statistically significant difference in cost between the lesion cohorts, with the lower costs being seen in those with only one lesion treated $(P=0.0014)$. This effect also holds when evaluating the number of lesions as a continuous covariate/ predictor in the linear regression model $(P=0.0076)$.

There were six OAS and two BA subjects with multiple lesions. Among subjects with one lesion treated (the majority of subjects), similar average costs were observed in the two arms. In an analysis where lesion cohort is included as a predictor in the model, we saw less evidence that a difference in average cost existed between arms (adjusted $P$-value for study arm $=0.1545$ ).

A linear regression model was fit to see whether there was a differential effect on cost between study arms across lesion cohorts (one lesion vs more than one lesion). There was statistical evidence of a possible interaction effect $(P=0.0360)$; thus, it is appropriate to report on subjects separately by lesion cohort. Figure 2 shows the average costs by study arm and lesion cohort, along with 95\% confidence intervals of the mean. With only two subjects in the BA-alone arm with more than one lesion, there are

Balloon (1 lesion)

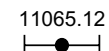

Balloon (>1 lesion)

10446.76

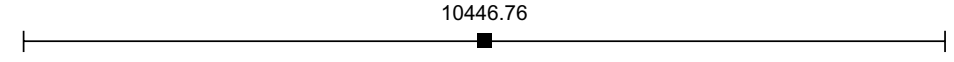

OAS (1 lesion)

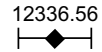

OAS (>1 lesion)

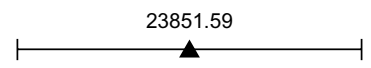

\begin{tabular}{|c|c|c|c|c|}
\hline 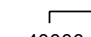 & 1 & 1 & 1 & ${ }_{40000}$ \\
\hline-40000 & -20000 & 0 & 20000 & 40000 \\
\hline
\end{tabular}

Figure 2 Average cost by study arm and lesion cohort (with $95 \%$ confidence intervals).

Abbreviation: OAS, orbital atherectomy system. 
insufficient data to get a reasonable estimate of cost in that group of subjects.

Excluding those subjects with more than one lesion, we see that there is no evidence of a statistical difference in cost between study arms $(P=0.4694)$.

\section{Cost-effectiveness comparison at 12 months}

The proportion of index procedure success and endpoints for the single-lesion population are presented in Figure 1. Note that the percentage of subjects who were free from acute adjunctive stenting was $94.7 \%(18 / 19)$ and $17.4 \%$ (4/23) for OAS+BA vs BA alone, respectively $(P<0.0001)$. Freedom from acute stenting, restenosis (due to TLR or TVR), or claudication at 1 year was $73.7 \%(14 / 19)$ and $8.7 \%(2 / 23)$ for OAS+BA vs BA alone, respectively. The estimated mean index procedure costs (for the single-lesion population) by procedural success and failure were US $\$ 10,516$ and US\$12,030, respectively, for OAS+BA and US\$6,951 and US\$9,860, respectively, for BA alone. In addition, the cost to treat restenosis was higher in BA alone vs OAS+BA (US\$13,734 vs US\$20,609) (Table 2).

Health utility values for procedural and 1-year outcomes are listed in Table 3. Subjects that required intervention to treat restenosis were assumed to have transitioned to lower QALY prior to treatment and experienced improved QoL post-procedure. Using these data, the mean costs for each outcome, and previously reported QALY, we approximated incremental cost of OAS+BA vs BA alone at 1 year as US\$549 and incremental QALY as 0.16 . This results in an incremental cost-effectiveness ratio of US\$3,441 (Table 4), well below the US\$50,000 threshold.

\section{Discussion}

For the subset of subjects with one lesion, COMPLIANCE $360^{\circ}$ reported higher (though not significantly) index

Table 2 Estimated procedural costs and future costs (singlelesion population)

\begin{tabular}{|c|c|c|c|}
\hline \multirow[t]{2}{*}{ Index procedure } & \multirow[t]{2}{*}{ Mean cost } & \multicolumn{2}{|l|}{ Range } \\
\hline & & Minimum & Maximum \\
\hline OAS+BA success & $\$ 10,516$ & $\$ 5,390$ & $\$ 21,867$ \\
\hline OAS+BA failure & $\$ 12,030$ & $\$ 12,030$ & $\$ 12,030$ \\
\hline BA success & $\$ 6,951$ & $\$ 5,257$ & $\$ 9,468$ \\
\hline BA failure & $\$ 9,860$ & $\$ 6,529$ & $\$ 31,077$ \\
\hline \multicolumn{4}{|c|}{ I Year - cost to treat restiosis } \\
\hline OAS revascularization & $\$ 13,735$ & $\$ 9,491$ & $\$ 18,254$ \\
\hline BA revascularization & $\$ 20,609$ & $\$ 19,808$ & $\$ 2 I, 4 I I$ \\
\hline
\end{tabular}

Note: Dollar values are in USD.

Abbreviations: BA, balloon angioplasty; OAS, orbital atherectomy system.
Table 3 Health utility values for quality-adjusted life year analysis (single-lesion population)

\begin{tabular}{llll}
\hline Outcome & $\begin{array}{l}\text { Health utility } \\
\text { value }\end{array}$ & Range & \\
\cline { 3 - 4 } & & Minimum & Maximum \\
\hline Procedure success & $0.8069^{19,20}$ & 0.702 & 0.912 \\
Procedure failure & $0.7328^{19}$ & $\mathrm{NR}$ & $\mathrm{NR}$ \\
$\begin{array}{l}\text { Restenosis } \\
\text { (limb ischemia) }\end{array}$ & $0.446^{21}$ & 0.150 & 0.550 \\
Claudication & 0.71 & & \\
\hline
\end{tabular}

Abbreviation: NR, not reported.

procedure costs for $\mathrm{OAS}+\mathrm{BA}$ compared with $\mathrm{BA}$ alone. When effectiveness is measured in terms of restenosis and/or subsequent TLR/TVR, OAS+BA and BA alone were comparable in terms of outcomes at 12 months. When freedom from acute adjunctive stenting was considered as an additional effectiveness measure, OAS+BA compared with BA alone had superior cost-effectiveness, with an expected cost of US\$3,441 per QALY gained to achieve an additional patient in whom acute adjunctive stenting was not required.

A recent study comparing directional atherectomy using the SilverHawk device (Covidien, Plymouth, MN, USA) to PTA to treat femoropopliteal disease reported similar procedural supply costs and overall hospital costs for both treatments with no significant difference in complication rates between the two groups, concluding that the choice of interventional procedure should depend on the operator's preference, skill, and experience. ${ }^{16}$ Safley et al reported that more stents were used in the PTA group compared with the SilverHawk group ( $1.5 \pm 0.8$ vs $0.2 \pm 0.5, P<0.001)$, a similar rate to that in COMPLIANCE $360^{\circ} .{ }^{16}$ Complications occurred at a somewhat higher rate in the SilverHawk study than the present study. In both studies, differences in device costs (atherectomy vs balloon) were offset by the additional cost of stenting as salvage therapy. Long-term health, imputed QoL, and economic outcomes were not assessed by Safley et al; ${ }^{16}$ therefore, COMPLIANCE $360^{\circ}$ is the first atherectomy study to examine cost-effectiveness explicitly.

Although overall patency outcomes were similar between treatments at 1 year, the two treatment arms of the study essentially resulted in two distinct outcomes: ISR vs restenosis without stenting. Currently, ISR remains an intractable problem with no approved and/or optimal treatments available, while OAS+BA may offer more straightforward revascularization options using currently approved existing technologies. Since the BA-alone group in COMPLIANCE $360^{\circ}$ led to primary stent placement in the majority of subjects (84\%) compared with just $8 \%$ of subjects receiving atherectomy, a key outcome of this study is that future treatment 
Table 4 Incremental cost-effectiveness ratio (single-lesion population)

\begin{tabular}{llllll}
\hline Treatment & Costs & QALY & Incremental costs & Incremental QALY & ICER \\
\hline BA & $\$ 12,939$ & 2.17 & & \\
OAS & $\$ 13,487$ & 2.33 & $\$ 549$ & 0.16 & $\$ 3,441$ \\
\hline
\end{tabular}

Note: Dollar values are in USD.

Abbreviations: BA, balloon angioplasty; ICER, incremental cost-effectiveness ration; OAS, orbital atherectomy system; QALY, quality-adjusted life year.

options were preserved for the majority of subjects treated with atherectomy.

Preservation of treatment options translates into increased cost for reintervention as observed in the BA-alone group compared with the OAS+BA group. Although the number of reinterventions was small, the BA group experienced an average cost of nearly US\$7,000 higher for those interventions. In addition, QALY and subsequent cost-effectiveness of OAS+BA demonstrate the benefit of maintaining treatment options. This is consistent with previous findings that stenting leads to more stenting and that those subsequent procedures require more devices and lead to increased costs. ${ }^{17,18}$

While the 1-year restenosis rate for treating calcific occlusive femoropopliteal disease with atherectomy and BA was similar to BA with provisional stenting, the atherectomybased approach may be more desirable given the high incidence of stenting in the angioplasty arm and the lack of a satisfactory treatment strategy for treating stent restenosis, especially diffuse pattern stent restenosis. The data from this study provide the opportunity to design future studies with the power necessary to draw more definitive conclusions. This study demonstrates that cost of the device alone should not be the deciding factor in selection of a vascular interventional treatment strategy but rather the overall cost of care, longerterm clinical outcomes, and consideration of the limitations and prognoses of salvage therapy.

\section{Limitations}

There are a number of limitations to the health economics analysis, including small sample size and limited cost data captured. The following data were not collected in the study: cost of the physician fee for the index procedure; other post-hospital discharge MRU data, including physician office visits and medications; and patient-reported outcomes such as activities of daily living and QoL. In addition, not all costs associated with rehospitalizations were directly reported via UB-04s. UB-04s were available for five vascular-related rehospitalizations. The additional event costs were estimated based on hospital-specific CCRs. Lastly, longer-term follow-up data and analysis is required to effectively analyze costs and benefits to the patient and society. Additional studies are warranted to fully understand the effect orbital atherectomy has on health outcomes and economics.

\section{Conclusion}

PAD remains a common and costly disorder, and given an aging population and increasing prevalence of diabetes, the treatment of PAD in these patients will have an expanding impact on clinical and economic resources. We found in this study that the index procedure costs and cost-effectiveness to 1 year were comparable for OAS+BA vs BA alone. These results provide compelling short-term health and economic data supporting the use of atherectomy in treatment of calcified femoropopliteal lesions, a longstanding challenge for PAD interventionalists. Additional cost-effectiveness analysis of PAD interventions is needed and should be included in all future peripheral vascular device trials.

\section{Acknowledgments}

The authors acknowledge the contributions of Katherine Kumar, PhD, and Brad J Martinsen, PhD, of Cardiovascular Systems, Inc., who edited and critically reviewed this manuscript. Lisa Thackeray of NAMSA provided statistical guidance and input.

\section{Author contributions}

Barry Weinstock and Raymond Dattilo, provided medical analysis, wrote/edited the manuscript, gave final approval of the submitted manuscript, and agree to be accountable for all aspects of the work. Tiffini Diage designed and executed economic analysis, wrote/edited the manuscript, gave final approval of the submitted manuscript, and agrees to be accountable for all aspects of the work.

\section{Disclosure}

All authors have served as consultants for Cardiovascular Systems, Inc. The authors have no other conflicts of interest in this work.

\section{References}

1. Norgren L, Hiatt WR, Dormandy JA, et al. Inter-Society Consensus for the Management of Peripheral Arterial Disease (TASC II). Eur J Vasc Endovasc Surg. 2007;33 Suppl 1:S1-S75. 
2. Roger VL, Go AS, Lloyd-Jones DM, et al. Heart disease and stroke statistics - 2011 update: a report from the American Heart Association. Circulation. 2011;123(4):e18-e209.

3. Hirsch AT, Haskal ZJ, Hertzer NR, et al. ACC/AHA 2005 guidelines for the management of patients with peripheral arterial disease (lower extremity, renal, mesenteric, and abdominal aortic): executive summary a collaborative report from the American Association for Vascular Surgery/Society for Vascular Surgery, Society for Cardiovascular Angiography and Interventions, Society for Vascular Medicine and Biology, Society of Interventional Radiology, and the ACC/AHA Task Force on Practice Guidelines (Writing Committee to Develop Guidelines for the Management of Patients With Peripheral Arterial Disease) endorsed by the American Association of Cardiovascular and Pulmonary Rehabilitation; National Heart, Lung, and Blood Institute; Society for Vascular Nursing; TransAtlantic Inter-Society Consensus; and Vascular Disease Foundation. J Am Coll Cardiol. 2006;47(6):1239-1312.

4. Almahameed A, Bhatt DL. Contemporary management of peripheral arterial disease: III. Endovascular and surgical management. Cleve Clin J Med. 2006;73 Suppl 4:S45-S51.

5. Bishop PD, Feiten LE, Ouriel K, et al. Arterial calcification increases in distal arteries in patients with peripheral arterial disease. Ann Vasc Surg. 2008;22(6):799-805.

6. Fitzgerald PJ, Ports TA, Yock PG. Contribution of localized calcium deposits to dissection after angioplasty. An observational study using intravascular ultrasound. Circulation. 1992;86(1):64-70.

7. Scheinert D, Scheinert S, Sax J, et al. Prevalence and clinical impact of stent fractures after femoropopliteal stenting. J Am Coll Cardiol. 2005;45(2):312-315.

8. Iida O, Nanto S, Uematsu M, Ikeoka K, Okamoto S, Nagata S. Influence of stent fracture on the long-term patency in the femoro-popliteal artery: experience of 4 years. JACC Cardiovasc Interv. 2009;2(7):665-671.

9. Korabathina R, Mody KP, Yu J, Han SY, Patel R, Staniloae CS. Orbital atherectomy for symptomatic lower extremity disease. Catheter Cardiovasc Interv. 2010;76(3):326-332.

10. Safian RD, Niazi K, Runyon JP, et al. Orbital atherectomy for infrapopliteal disease: device concept and outcome data for the OASIS trial. Catheter Cardiovasc Interv. 2009;73(3):406-412.
11. Makam P. Use of orbital atherectomy treatment in a high-volume clinical practice modifies non-compliant plaque to deliver durable long-term results. J Invasive Cardiol. 2013;25(2):85-88.

12. Shammas NW, Lam R, Mustapha J, et al. Comparison of orbital atherectomy plus balloon angioplasty vs balloon angioplasty alone in patients with critical limb ischemia: results of the CALCIUM 360 randomized pilot trial. J Endovasc Ther. 2012;19(4):480-488.

13. Rosamond W, Flegal K, Furie K, et al. Heart disease and stroke statistics - 2008 update: a report from the American Heart Association Statistics Committee and Stroke Statistics Subcommittee. Circulation. 2008;117(4):e25-e146.

14. Hirsch AT, Hartman L, Town RJ, Virnig BA. National health care costs of peripheral arterial disease in the Medicare population. Vasc Med. 2008;13(3):209-215.

15. Dattilo R. The COMPLIANCE $360^{\circ}$ Trial: the principal investigator of the COMPLIANCE $360^{\circ}$ trial discusses its rationale, study design, and potential therapeutic implications. Endovascular Today. Mar 2010.

16. Safley DM, Lindsey JB, Robertus K, et al. In-hospital outcomes and cost comparison of femoropopliteal reopening strategies. Cardiovasc Revasc Med. 2011;12(5):292-298.

17. Burket MW. Restinosis and the New Health Care Economy. Endovascular Today. 2013:42-48.

18. Robinson WP 3rd, Nguyen LL, Bafford R, Belkin M. Results of secondtime angioplasty and stenting for femoropopliteal occlusive disease and factors affecting outcomes. J Vasc Surg. 2011;53(3):651-657.

19. Egberg L, Mattiasson AC, Ljungstrom KG, Styrud J. Health-related quality of life in patients with peripheral arterial disease undergoing percutaneous transluminal angioplasty: a prospective one-year follow-up. J Vasc Nurs. 2010;28(2):72-77.

20. Kalbaugh CA, Taylor SM, Blackhurst DW, Dellinger MB, Trent EA, Youkey JR. One-year prospective quality-of-life outcomes in patients treated with angioplasty for symptomatic peripheral arterial disease. J Vasc Surg. 2006;44(2):296-302; discussion 302-293.

21. de Vries SO, Visser K, de Vries JA, Wong JB, Donaldson MC, Hunink MG. Intermittent claudication: cost-effectiveness of revascularization versus exercise therapy. Radiology. 2002;222(1): $25-36$.
ClinicoEconomics and Outcomes Research

\section{Publish your work in this journal}

ClinicoEconomics \& Outcomes Research is an international, peerreviewed open-access journal focusing on Health Technology Assessment, Pharmacoeconomics and Outcomes Research in the areas of diagnosis, medical devices, and clinical, surgical and pharmacological intervention. The economic impact of health policy and health systems

\section{Dovepress}

organization also constitute important areas of coverage. The manuscript management system is completely online and includes a very quick and fair peer-review system, which is all easy to use. Visit http://www.dovepress.com/testimonials.php to read real quotes from published authors. 